\title{
Effect of fecal microbiota transplantation route of administration on gut colonization and host response in preterm pigs
}

\author{
Anders Brunse $\mathbb{1}^{1} \cdot$ Lena Martin $^{1,2} \cdot$ Torben Sølbeck Rasmussen $^{3} \cdot$ Lars Christensen $^{1} \cdot$ Malene Skovsted Cilieborg $^{1}$ • \\ Maria Wiese ${ }^{3} \cdot$ Bekzod Khakimov $^{3} \cdot$ Robert Pieper $^{2} \cdot$ Dennis Sandris Nielsen $^{3} \cdot$ Per Torp Sangild $^{1}$ • \\ Thomas Thymann (1) $^{1}$
}

Received: 18 May 2018 / Revised: 26 September 2018 / Accepted: 4 October 2018 / Published online: 26 October 2018

(c) International Society for Microbial Ecology 2018

\begin{abstract}
This study examined gut colonization patterns and host responses to fecal microbiota transplantation (FMT) by different administration routes after preterm birth. In two separate experiments, cesarean-delivered, preterm pigs were administered combined oral + rectal, or exclusively rectal donor feces, and compared with saline controls. After 5 days, stomach and colon bacterial compositions were determined by $16 \mathrm{~S}$ rRNA gene amplicon sequencing, and organic acid metabolites measured. Further, gut pathology, mucosa bacterial adherence, and goblet cell density were assessed. FMT increased the relative abundance of obligate anaerobes in the colon without affecting total bacterial load. Bacteroides colonized recipients despite low abundance in the donor feces, whereas highly abundant Prevotella and Ruminococcaceae did not. Further, FMT changed carbohydrate metabolism from lactate to propionate production thereby increasing colonic $\mathrm{pH}$. Besides, FMT preserved goblet cell mucin stores and reduced necrotizing enterocolitis incidence. Only rectal FMT increased the stomachto-colon $\mathrm{pH}$ gradient and resistance to mucosa bacterial adhesion. Conversely, oral + rectal FMT increased bacterial adhesion, internal organ colonization, and overall mortality. Our results uncovered distinctions in bacterial colonization patterns along the gastrointestinal tract, as well as host tolerability between oral and rectal FMT administration in preterm newborns. Besides, FMT showed the potential to prevent necrotizing enterocolitis.
\end{abstract}

\section{Introduction}

Necrotizing enterocolitis (NEC) affects $5-15 \%$ of infants born before 32 weeks' gestation and is associated with high mortality (up to $30 \%$ ) and chronic morbidities such as impaired neurodevelopment [1]. Preterm infants are

Electronic supplementary material The online version of this article (https://doi.org/10.1038/s41396-018-0301-z) contains supplementary material, which is available to authorized users.

$\triangle$ Thomas Thymann

thomas.thymann@sund.ku.dk

1 Department of Veterinary and Animal Sciences, Comparative Pediatrics and Nutrition, Faculty of Health and Medical Sciences, University of Copenhagen, Frederiksberg, Denmark

2 Institute of Animal Nutrition, Freie Universität Berlin, Berlin, Germany

3 Department of Food Science, Faculty of Science, University of Copenhagen, Frederiksberg, Denmark predisposed to NEC due to intestinal immaturity, characterized by decreased nutrient digestion and absorption, dysmotility, impaired intestinal barrier function, and an immature mucosal immune system. In preterm infants, aggressive enteral feeding with infant formula may disturb the gut microbial colonization and promote a dysbiosis characterized by low bacterial species diversity and malfermentation, compromising barrier function and leading to intestinal inflammation and injury [2].

Gut colonization and establishment of a stable microbial community is an orchestrated event, where early dominance of facultative anaerobes is succeeded by mainly obligate anaerobes, and preterm infants take longer time to acquire an obligate anaerobic dominated gut microbiota [3]. Facultative anaerobes (e.g., Enterococcaceae, Enterobacteriaceae) are increased and obligate anaerobes (e.g., Bifidobacterium, Bacteroides) decreased in abundance, while propionate production is decreased in preterm versus term infants during the first 1-3 months of life [4]. Several papers report characteristic NEC-related patterns in the gut microbial composition of preterm infants [5-7], but a high 
degree of temporal and geographical variation indicates that a uniform NEC microbiota does not exist [8]. The few consistent conclusions from a data synthesis of gut microbiota profiling in preterm newborns include increased abundance of Proteobacteria and decreased abundances of Firmicutes and Bacteroidetes in relation with NEC [9]. In a recent study using serial fecal samples from preterm infants with and without NEC, the gut microbiota separated into six distinct clusters [10]. Five of six clusters were characterized by a single or few dominant facultative anaerobic genera (Klebsiella, Escherichia, Enterococcus, or Staphylococcus), whereas the last cluster had a more diverse composition. NEC infants experienced more frequent cluster transitions and occupied any of the five dysbiotic clusters, whereas the sixth cluster had no NEC cases, suggesting that microbiota instability rather than any particular composition may predispose to NEC development.

Probiotics ["live microorganisms which when administered in adequate amounts confer a health benefit on the host" [11]] is a proposed bacterial treatment in preterm infants to prevent NEC. A recent meta-analysis of 24 probiotic trials for NEC prevention strongly recommends implementation of probiotics treatment in routine care of preterm infants [12]. However, there is inconsistency with regards to the choice of probiotics strain(s) [13]. Fecal microbiota transplantation (FMT, "the administration of fecal matter containing distal gut microbiota from a healthy donor") has recently gained interest due to efficient correction of gut microbial dysbiosis and clinical improvement in several adult patient groups, most notably recurrent Clostridium difficile infection [14]. FMT is administered via either an upper or lower gastrointestinal route, or a combination [14]. In preterm infants, a bacterial therapy containing a balanced and diverse microbiota from a healthy donor may be more efficient at stabilizing the newborn gut microbiota relative to conventional probiotics with potential implications for NEC development.

Formula-fed preterm pigs recapitulate most clinical and pathological features of NEC including feeding intolerance, abdominal distention, gut pathology ranging from hyperemia and local hemorrhage to pneumatosis intestinalis, transmural necrosis and portal venous gas, often progressing to peritonitis, sepsis and shock [15]. Like in infants, NEC development is microbiota dependent with no evidence of NEC under germ-free conditions [15], and limited NEC following enteral antibiotics treatment [16]. Administration of probiotic mixtures to preterm newborn pigs has produced conflicting results, showing either a moderate benefit or a clear increase in NEC sensitivity [17, 18]. Preterm neonates may be particularly sensitive to gut bacterial overgrowth just after birth, and a proper composition, dose and administration route of the bacterial inoculum may be critical to avoid excessive bacterial stimulation of the small intestine.

We hypothesized that FMT would affect the bacterial colonization of preterm neonates, improve mucosal barrier structure and protect against NEC. Using preterm pigs as models for preterm infants, we performed two separate experiments investigating combined oral + rectal FMT administration (experiment 1 ), followed by rectal administration alone (experiment 2) with donor feces obtained from 10-day-old healthy, term, sow-reared piglets.

\section{Materials and methods}

\section{Animal experimental procedures}

One hundred thirty-three live piglets (Danish landrace $x$ Large White $\times$ Duroc) were delivered by cesarean section at $90 \%$ gestation $(106 \pm 1 \mathrm{~d})$ from six healthy sows. Animals were immediately moved to and housed individually in preheated incubators $\left(37^{\circ} \mathrm{C}\right)$ with oxygen supply (1-2 l/ $\min$ ) for the first $12 \mathrm{~h}$ and fitted with orogastric and umbilical arterial catheters for gavage feeding and vascular access (6 and 4 Fr size, respectively, Portex, Kent, UK), as previously described [19]. The animals were gavage fed every $3 \mathrm{~h}$ with increasing volumes of infant formula $(24,40$, and $120 \mathrm{ml} / \mathrm{kg} / \mathrm{d}$ on days 1,2 , and 3-5, respectively. Infant formula recipe presented in Suppl. table 1) and supplemented for the first 2 days with parenteral nutrition (4-6 ml/kg/h, Kabiven, Vamin, Fresenius-Kabi, Bad Homburg, Germany).

\section{FMT}

We collected colon luminal content from five 10-day-old, healthy appearing suckling piglets. The content was pooled and homogenized, diluted 1:1 in $20 \%$ sterile glycerol and frozen. Before inoculation, fecal samples were diluted in sterile saline to a working concentration of $0.05 \mathrm{~g} / \mathrm{ml}$ and filtered through a 70- $\mathrm{m}$ cell strainer. In experiment 1 , preterm pigs received combined oral + rectal administration of FMT. Pigs were blocked by gender and birth weight and randomly allocated to receive oral and rectal FMT inoculations $(0.025 \mathrm{~g}$ feces per inoculum in $0.5 \mathrm{ml}$ sterile saline) twice daily, amounting to $0.1 \mathrm{~g}$ feces per day for the first 2 days of life (FMT, $n=41$ ), or similar volumes of control saline ( $\mathrm{CON}, n=42)$. The oral inoculate was administered via the orogastric feeding tube, and the rectal inoculate was administered via a soft rectal probe (6 Fr size) placed $50 \mathrm{~mm}$ into the rectum. In experiment 2, preterm pigs received FMT only by rectal administration. Animals were blocked by gender and birth weight and randomly allocated to 
receive rectal FMT $(0.025 \mathrm{~g}$ feces per inoculum in $0.5 \mathrm{ml}$ sterile saline) twice daily, to reach $0.05 \mathrm{~g}$ feces per day during the first 2 days (FMT, $n=22$ ), with controls receiving similar volumes of saline $(\mathrm{CON}, n=23)$.

\section{Clinical observations and in vivo functional tests}

Animals were continuously monitored by experienced personnel and immediately euthanized if presenting with clinical signs of systemic illness. Motor activity was captured by continuous infrared video surveillance connected to a motion detection software (PigLWin, Ellegaard Systems, Faaborg, Denmark). Hexose absorptive capacity was assessed on day 4 by gastric delivery of a $10 \%$ galactose bolus $(8 \mathrm{ml} / \mathrm{kg})$ and plasma galactose recovery after $20 \mathrm{~min}$. Intestinal permeability was assessed on day 5 by gastric delivery of a $5 / 5 \%$ lactulose $/$ mannitol bolus $(15 \mathrm{ml} / \mathrm{kg})$ and urinary recovery after $3 \mathrm{~h}$. Plasma galactose and urinary lactulose and mannitol concentrations were measured as previously described [16]. Experimental procedures conducted during the animal experiment were approved by the Danish Animal Experiments Inspectorate (license number, 2014-15-0201-00418), which is in accordance with the guidelines from Directive 2010/63/EU of the European Parliament.

\section{Euthanasia, tissue collection, and NEC evaluation}

Animals were euthanized on day 5 by intracardiac injection of sodium-pentobarbital, after a blood sample had been drawn by cardiac puncture for hematology and clinical biochemistry. Aseptic biopsies of the spleen and liver were collected for bacteriology. Urine was collected by cystocentesis for measurement of lactulose and mannitol. Abdominal organs were excised, and macroscopic lesions of stomach, proximal, mid and distal small intestine, and colon were graded according to a previously validated NEC scoring system (Fig. 1a) [20]. NEC was defined as the presence of macroscopic lesions (score 3-6) in at least one gastrointestinal segment. Stomach and colon luminal contents were snap-frozen for analysis of bacterial composition, $\mathrm{pH}$ measurement, and organic acid analysis. Small intestine and colon tissues were snap-frozen or immersed in $4 \%$ paraformaldehyde for determination of brush-border enzyme activities, gene expression, cytokine analyses, and histology.

\section{Gut microbiota analysis and organic acids}

Stomach and colon luminal content collected at the time of euthanasia, as well as a single aliquot of donor feces were homogenized before DNA extraction using Qiagen DNeasy PowerSoil kit (Qiagen AB, Sollentuna, Sweden). DNA concentration and purity were assessed using a NanoDrop 1000 spectrophotometer (Thermo Fisher Scientific, Waltham, MA, USA). DNA samples were subjected to tagencoded 16S ribosomal RNA gene MiSeq-based highthroughput sequencing (Illumina, San Diego, CA, USA), with sequencing library preparation steps conducted as previously described [22]. The V3 region ( $190 \mathrm{bp})$ of the 16S rRNA gene was amplified using primers compatible with Nextera Index Kit (Illumina): NXt_388_F: 5'TCGTCGGCAGCGTCAGATGTGTATAAGAGACAGACWCCTACGGGWGGCAGCAG-3' and NXt_518_R: 5'GTCTCGTGGGCTCGGAGATGTGTATAAGAGACAGATTACCGCGGCTGCTGG-3' (Integrated DNA Technologies; Leuven, Belgium). PCR reactions containing $12 \mu \mathrm{l}$ AccuPrimeTM SuperMix II (Life Technologies, CA, USA), $0.5 \mu \mathrm{l}$ of each primer $(10 \mu \mathrm{M}), 5 \mu \mathrm{l}$ of genomic DNA $(\sim 1-$ $10 \mathrm{ng} / \mu \mathrm{l}$ ), and $2 \mu \mathrm{l}$ nuclease-free water to a total volume of $20 \mu \mathrm{l}$ were run on a SureCycler 8800 . Cycling conditions applied were: $95^{\circ} \mathrm{C}$ for $2 \mathrm{~min} ; 33$ cycles of $95^{\circ} \mathrm{C}$ for $15 \mathrm{~s}$, $55^{\circ} \mathrm{C}$ for $15 \mathrm{~s}$, and $68^{\circ} \mathrm{C}$ for $30 \mathrm{~s}$; followed by final step at $68^{\circ} \mathrm{C}$ for $5 \mathrm{~min}$. Agarose gel electrophoresis was performed to ensure that a 250-bp PCR product was obtained for each sample, except negative control. To incorporate primers with adapters and indexes, PCR reactions contained $12 \mu \mathrm{l}$ Phusion High-Fidelity PCR Master Mix (Thermo Fisher Scientific, MA, USA), $2 \mu$ corresponding P5 and P7 primer (Nextera Index Kit), $2 \mu \mathrm{l}$ PCR product, and nuclease-free water for a total volume of $25 \mu$ l. Cycling conditions were: $98^{\circ} \mathrm{C}$ for $1 \mathrm{~min} ; 13$ cycles of $98^{\circ} \mathrm{C}$ for $10 \mathrm{~s}, 55^{\circ} \mathrm{C}$ for $20 \mathrm{~s}$, and $72{ }^{\circ} \mathrm{C}$ for $20 \mathrm{~s}$; and $72{ }^{\circ} \mathrm{C}$ for $5 \mathrm{~min}$. The amplified fragments with adapters and tags were purified using AMPure XP beads (Beckman Coulter Genomic, CA, USA). Prior to library pooling, clean constructs were quantified using a Qubit Fluorometer (Invitrogen, Carlsbad, CA, USA) and mixed in approximately equal concentrations to ensure even representation of reads per sample. Each sample was represented with approximately $30 \mathrm{ng}$ DNA. Subsequently, 250-bp pair-ended MiSeq (Illumina, CA, USA) sequencing was performed according to the instructions of the manufacturer.

Raw MiSeq reads were processed by the IMNGS [21] pipeline (version 1.0 Build 1808), which is based on UPARSE [22] and USEARCH [23]. Allowed mismatches was set to 2 , minimum fastq quality score for trimming of unpaired reads to 3, max number of expected errors in paired sequences to 3 , and the minimum relative Operational Taxonomic Unit (OTU) abundance to $0.25 \%$. The analysis delivered 11,097,993 quality and chimera-checked reads for experiment 1 (average 147,973 $\pm 51,077$ reads per sample) and 6,895,769 reads for experiment 2 (average $137,915 \pm 45,310$ reads per sample). Samples with $<10,000$ reads were discarded. OTU's were clustered at $97 \%$ sequence identity. Alignment of IMNGS generated OTU 
Fig. 1 Clinical response to different FMT administration routes. a Representative autopsy photographs of pig stomach, intestine, and colon with various NEC lesion severities. The NEC lesion scoring system is defined as follows: (1) normal appearance; (2) mild hyperemia and/or edema; (3) extensive hyperemia, edema and/or minor hemorrhagic lesions; (4) extensive hemorrhagic lesions and pneumatosis intestinalis; (5) hemorrhagic lesions with local necrosis and pneumatosis intestinalis; (6) extensive hemorrhagic lesions, necrosis and pneumatosis intestinalis. b Five-day survival curves. c Continuous home-cage motor activity index. d Daily growth rate. e NEC incidence. NEC was defined as a lesion score of at least 3 in one or more gut segments (see above). ${ }^{*} p<0.05$, $* * p<0.01, * * * p<0.001$
A

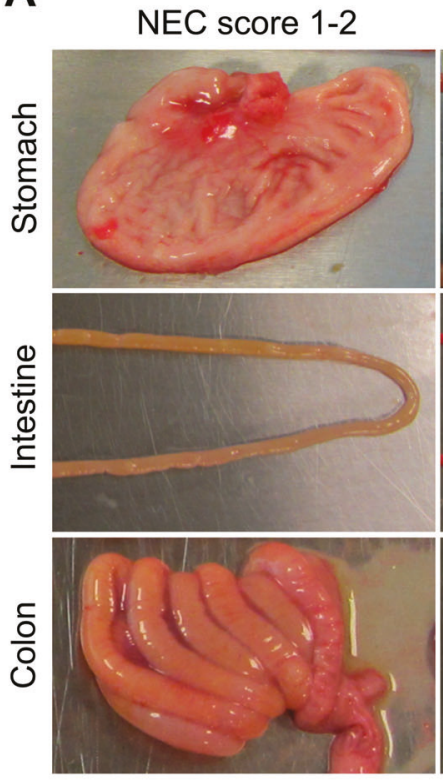

B

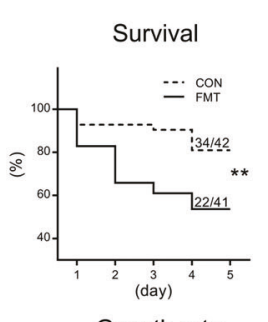

Oral + rectal FMT
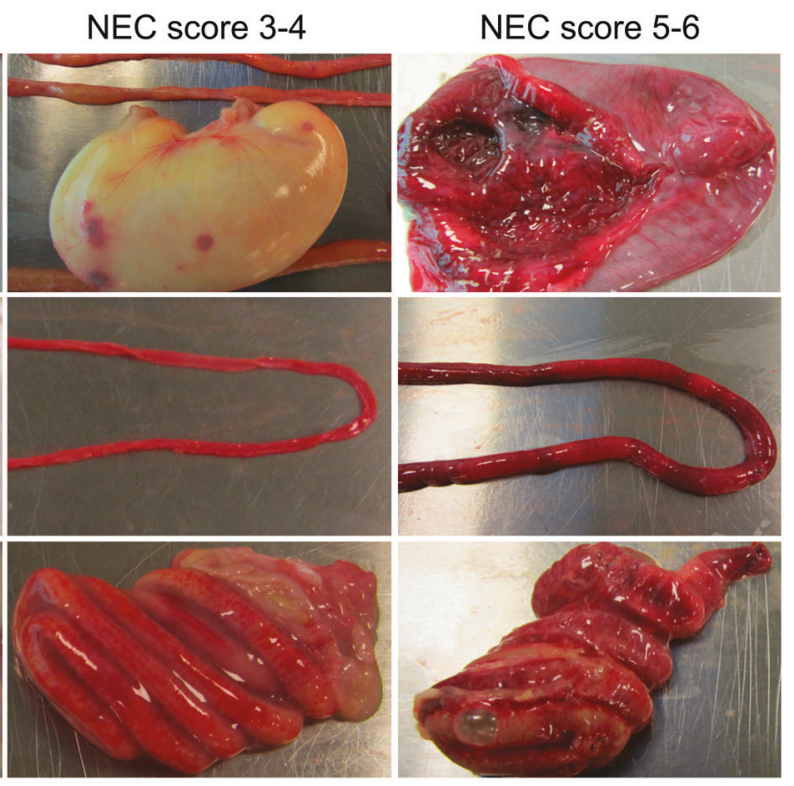

Rectal FMT

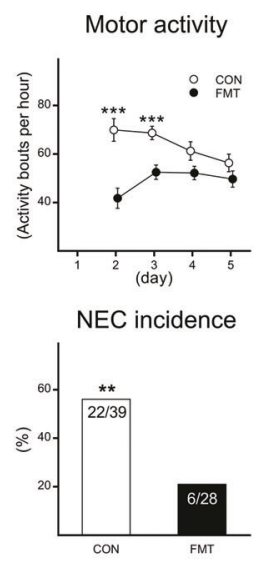

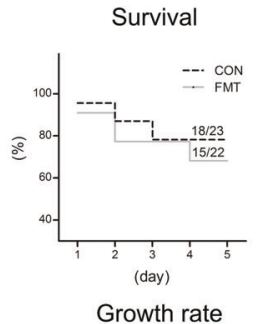

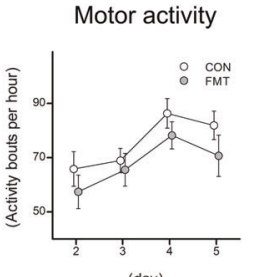

(day)

NEC incidence
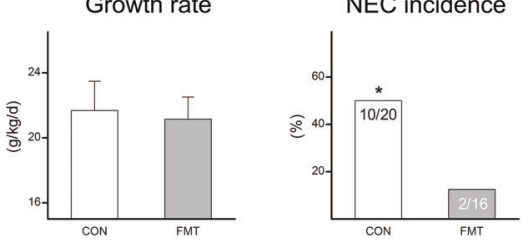

sequences with SINA version 1.2.11 [24] (default settings) was performed to obtain the highest possible level of taxonomy of each OTU. The R-based RHEA pipeline was executed for data analysis yielding rarefaction curves (Suppl. Figure 1), NMDS plots and taxonomic binning. Unweighted/weighted UniFrac [25] represented the phylogenetic beta-diversity analysis and the Shannon index represented the alpha diversity. Normalization of sequencing depth was done by rarefaction with RHEA (experiment 1: 46,026 reads and experiment 2: 49,737 reads) via division by the sum of sequences in a given sample and multiplication by the minimum sum across all samples. Data files processed by RHEA was imported to Quantitative Insight Into Microbial Ecology 2 [26] (2018.4 build 1525276946) to calculate group significance. The nonparametric Kruskal-Wallis and ANOSIM were used to calculate group significance for, respectively, alpha and beta diversity.

Functional prediction of colonic microbiota was performed using Phylogenetic Investigation of Communities by Reconstruction of Unobserved States v. 1.1.3 (PICRUSt) [27] by QIIME 1.9.1. PICRUSt formatted data were normalized with cumulative sum scaling (normalize_table.py) to adjust for sequence depth variation and summarized using the Kyoto Encyclopedia of Genes and Genomes (KEGG) orthology database. Only colon control and FMT samples were included in the PICRUSt analysis. Relative bacterial abundance and PICRUSt-data were analyzed using a Kruskal-Wallis test (group_significance.py) and considered noteworthy, if $q$-values (false discovery ratecorrected test values) were below 0.05 and abundances at least twofold different. Bacterial density in stomach and 
colon luminal content was estimated by quantitative realtime polymerase chain reaction, using $16 \mathrm{~S}$ rRNA universal primers and standard curves based on total extracted DNA from pure Escherichia coli $\mathrm{K}-12$ [28].

Gastric and colonic D- and L-lactate were analyzed by high performance liquid chromatography on an Agilent 1100 chromatograph equipped with a Phenomenex C18 $(4.0 \times 2.0 \mathrm{~mm})$ guard column followed by a Phenomenex Chirex 3126 (D)-penicillamine column $(150 \times 4.6 \mathrm{~mm})$ and a UV detector at $253 \mathrm{~nm}$. Short chain fatty acids (SCFAs) acetate, propionate, and butyrate were determined by gas chromatography on a polyethylene glycol column $(30 \mathrm{~m}$ by $530 \mu \mathrm{m}$ by $1.00 \mu \mathrm{m}$; HP-INNOWax) in an Agilent 6890 gas chromatography system with caproic acid as internal standard [29]. Gastric and colonic $\mathrm{pH}$ were measured in diluted and homogenized samples using a $\mathrm{pH}$-meter.

\section{Gut mucosal structure, function, and immune response}

Bacterial adhesion to small intestinal mucosa was visualized by fluorescence in situ hybridization (FISH) [16]. Sections were converted to digital images using an Axio Scan.Z1 fluorescence slide scanner (Zeiss Microscopy, Jena, Germany). Bacterial signal was quantified by image analysis using the Visiopharm software package (Visiopharm, Hørsholm, Denmark) and expressed as pixelated area. Villus morphometry, small intestinal brush-border enzyme activity, and colonic goblet cell density were measured as previously described [16]. Gene expression analysis of small intestinal tissue was performed in experiment 1 , using the same primer sequences for target (TNF, IFNG, IL6, IL8, IL10, IL17, TGFB1, PCNA, MUC1, MUC2, MUC5AC, MUC20, TLR2, TLR4, CLDN2, CLDN4, OCLN, TJP1) and reference genes (B2M,SDHA), as previously described [30]. Colonic cytokine protein levels were measured using porcine DuoSet ELISA kits (R\&D Systems Denmark, Abingdon, UK).

\section{Bacteriology, hematology, and clinical biochemistry}

Blood and organ bacteriology was performed in experiment 1. Blood and homogenized liver and spleen samples were plated on blood agar and incubated under aerobic and anaerobic conditions $\left(24 \mathrm{~h}, 37^{\circ} \mathrm{C}, 5 \% \mathrm{CO}_{2}\right)$. Colonizing bacteria were identified using matrix-assisted laser desorption-ionization time-of-flight mass spectrometry without enumeration. Hematology and clinical biochemistry were measured in EDTA-stabilized whole blood and serum respectively, using Advia 2120i Hematology and Advia 1800 Clinical Chemistry Systems (Siemens Healthcare $\mathrm{GmbH}$, Erlangen, Germany) on day 5, whereas serum C- reactive protein (CRP) was measured using a porcine DuoSet ELISA kit (R\&D Systems, Denmark).

\section{Statistics}

Survival rates were analyzed by log-rank test, NEC incidence by Fisher's exact test, and organ colonization incidence by Pearson's chi-squared test. Remaining endpoints were analyzed by linear mixed models, using Stata statistical package (v11, StataCorp, College Station, TX, US). Data are presented as means with standard error unless otherwise specified.

\section{Results}

\section{Experiment 1: oral + rectal FMT}

\section{Clinical observations}

Two animals could not be resuscitated after birth and died before randomization. Five-day survival was markedly reduced in the group administered oral + rectal FMT compared with controls (53 vs $81 \%, p<0.01, n=41-42$, Fig. 1b, left). This difference in survival arose from fewer FMT pigs surviving the first $48 \mathrm{~h}(66 \mathrm{vs} 93 \%, p<0.01)$, with no difference thereafter ( 81 vs $87 \%$ ). Deaths before 48 $\mathrm{h}$ were unrelated to gastrointestinal symptoms and no NEC lesions were observed at autopsy in these animals. FMT pigs had reduced motor activity compared with controls on days 2 and 3 (both $p<0.001, n=22-41$, Fig. 1b), while on days 4 and 5 the activity levels were similar. FMT animals surviving until day 5 showed reduced growth compared with control ( $p<0.05, n=20-34$, Fig. 1 b), but $60 \%$ relative reduction in NEC incidence ( 21 vs $56 \%, p<0.01, n=28$ 39, Fig. 1b). Across groups, lesions mainly occurred in the colon (FMT: $11 \%$ vs CON: $46 \%, p<0.01$ ) with fewer lesions in the stomach (4\%), proximal (10\%), middle (13\%), and distal (18\%) intestine.

\section{Gut microbial composition and metabolites}

Oral + rectal FMT affected the bacterial composition in stomach (unweighted UniFrac, $R=0.464, \quad p<0.001$; weighted UniFrac, $R=0.067, \quad p<0.05)$ and colon (unweighted UniFrac, $R=0.368, p<0.01$; weighted UniFrac, $R=0.141, p<0.05)$ when compared with control (Fig. 2a, left). The treatment increased bacterial Shannon diversity in the stomach $(p<0.001$, Fig. $2 \mathrm{~b}$, left) but not colon, which was substantially lower than that of the donor fecal microbiota. Lactobacillus was the dominant genus in stomach content in both FMT and CON, but relative 
A

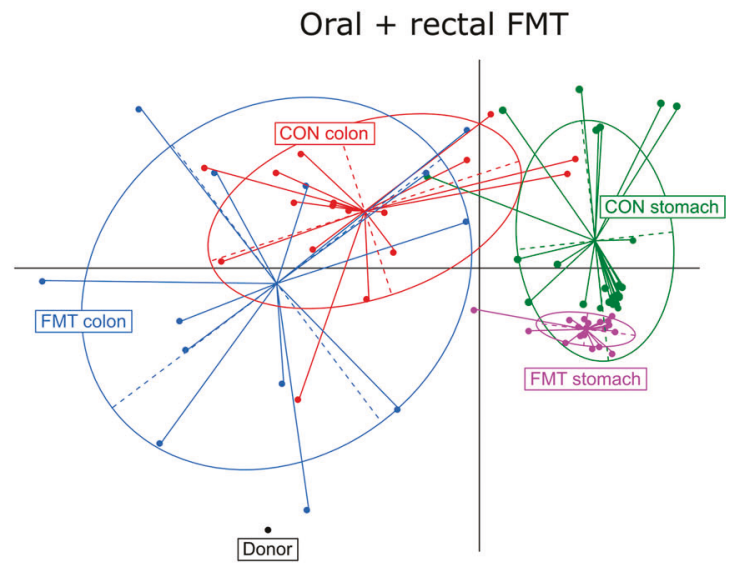

\section{Beta diversity}

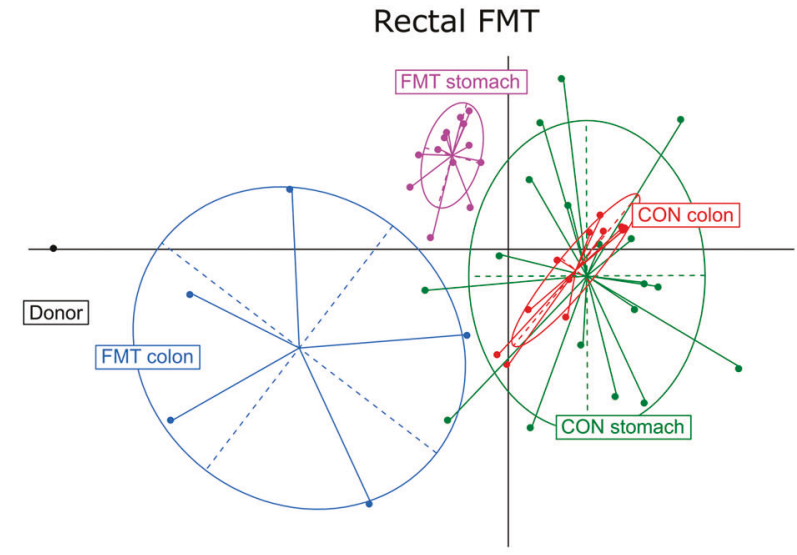

B

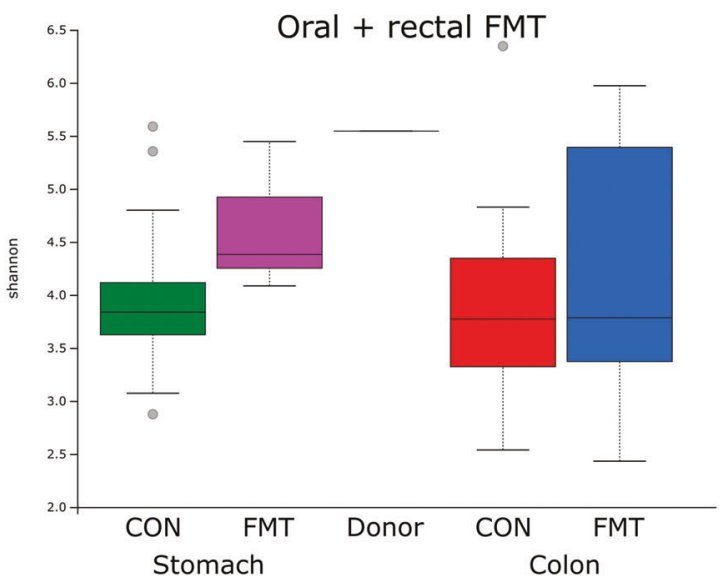

Alpha diversity

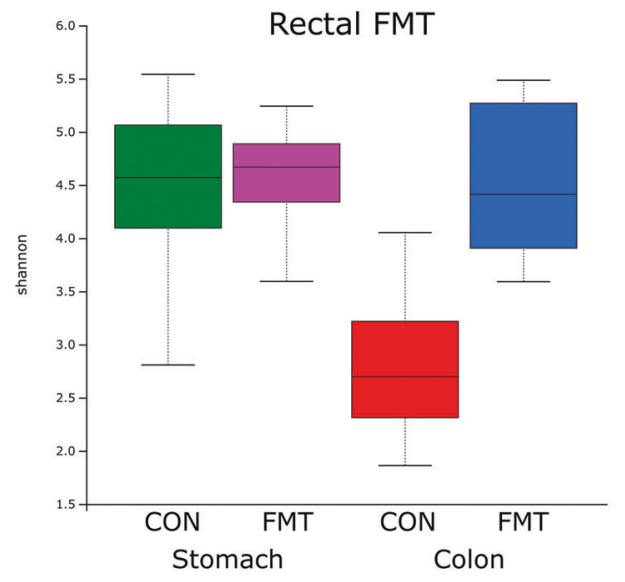

Rectal FMT

Bacterial taxonomy

C

Oral + rectal FMT

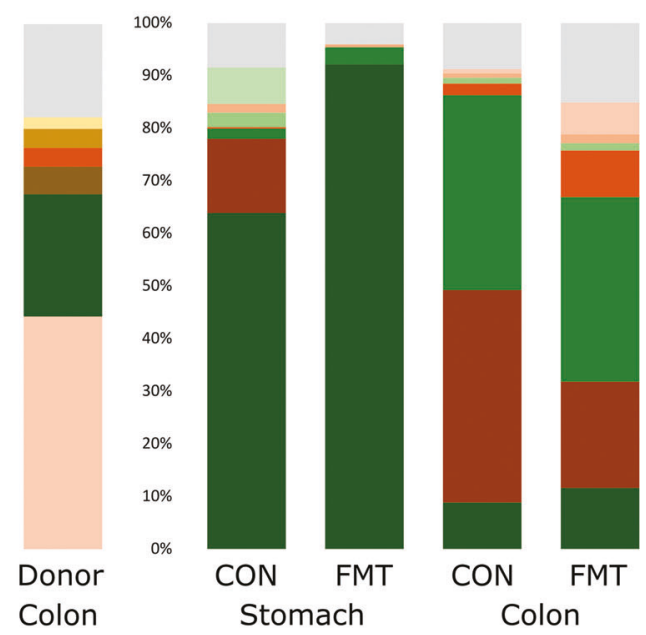

$100 \%$

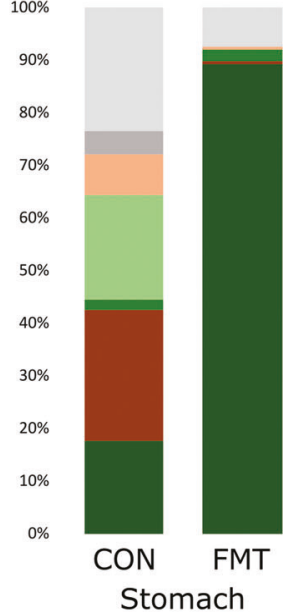

Rectal FMT

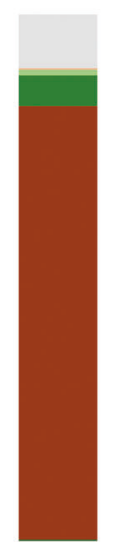

CON FMT

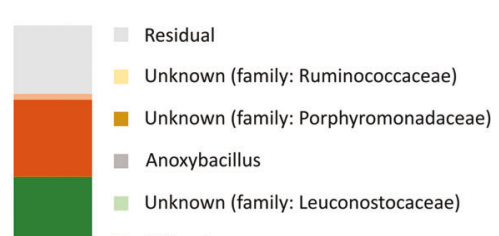

- RC9 gut group

In Prevotella

- Streptococcus

- Staphylococcus

- Bacteroides

- Escherichia

- Enterococcus
Fig. 2 Gut microbiota composition after different FMT administration routes. a Unweighted UniFrac metrics-based measure of beta diversity visualized using non-metric multidimensional scaling. b Shannon index as a measure of alpha diversity presented as box- and-whisker plots. c Relative bacterial abundances in stomach and colon luminal contents, as well as donor feces presented as stacked bar graphs showing dominant taxa (mean abundance $>1 \%$ ) at genus level 
abundances of Enterococcus (14.3 vs $0.10 \%, p<0.001)$ and an unclassified genus within Leuconostoceae (6.98 vs $0.10 \%, p<0.001)$ were higher in CON pigs. In colon, FMT reduced the relative abundance of Enterococcus (20.2 vs $40.3 \%, p<0.05$, Fig. 2c) accompanied by increased abundances of obligate anaerobic genera Bacteroides (9.04 vs $2.17, p<0.05)$ and Prevotella (5.88 vs $0.85 \%, p<0.01)$. Sample-level taxonomy charts are provided for assessment of within-group variation (Suppl. Figure 2).

No significant changes to any functional pathways was identified by PICRUSt analysis.

Oral + rectal FMT led to a 10-fold increase in stomach bacterial density ( $p<0.001$, Fig. 3a, upper), as well as a threefold increase in total measured organic acids compared with control $(45.8 \pm 4.6$ vs $15.8 \pm 2.3 \mu \mathrm{mol} / \mathrm{g}, p<0.001)$. This was mainly due to an increased lactate level, but also acetate and butyrate were significantly increased (all $p<$ 0.001 , Fig. 3a) and accompanied by an increase in stomach pH $(p<0.001$, Fig. 3a). In the colon, total bacterial density was not affected by FMT treatment (Fig. 3a). However, total colonic organic acids were decreased inFMT pigs compared with CON $(68.5 \pm 4.4$ vs $41.2 \pm 6.3 \mu \mathrm{mol} / \mathrm{g}, p<$ $0.001)$ due to decreased levels of the most abundant metabolite lactate $(p<0.001$, Fig. 3a), whereas propionate levels were fourfold higher after FMT treatment $(p<0.05)$. Additionally, the $\mathrm{pH}$ of colon luminal content was significantly higher in FMT pigs ( $p<0.01$, Fig. 3 a).

\section{Gut mucosal structure, function, and immune response}

In formula-fed preterm pigs, abundant bacteria were adhering to the villi along the entire small intestine, indicating mucosal barrier disruption (Fig. 3b, left). In oral + rectal FMT-treated animals, the abundance of adhering bacteria measured by quantitative FISH staining was higher than in controls ( $p<0.05$, Fig. 3b, upper right), although the mucosal immune response appeared to be reduced (Fig. 4a). Thus, the expression level of the microbial antigen recognition receptor $T L R 2$ was increased, whereas expression of proinflammatory TLR4 was decreased in the small intestine of FMT animals relative to NEC-afflicted controls (both $p<$ 0.05 , Fig. 4a). Besides, the expression of several cytokines including $T N F, I L 6, I L 8$, and ILI7A were increased in controls with NEC only (all $p<0.05$, Fig. $4 a$ ). Moreover, proximal small intestinal villi were longer, and lactase and dipeptidyl peptidase-IV activities increased in FMT pigs compared with controls (all $p<0.05$, Suppl. table S2). Intestinal permeability (urinary lactulose-mannitol ratio, pooled median: 0.15 [interquartile range (IQR): 0.19], $n=$ 16-22) and hexose absorption (plasma galactose, pooled mean: 97.1 [SD: 75] $\mu \mathrm{M}, n=22-34$ ) were not affected.

The density of mucin-containing colonic goblet cells was generally low following formula feeding, and with total depletion of the mucin pool occurring locally (Fig. 4c, left). In oral + rectal FMT-treated animals, the mucin density in colon was significantly higher than controls $(p<0.05$, Fig. $4 c$, right), possibly due to the higher NEC burden of CON animals, as NEC severity was inversely correlated with mucin density $(0.8 \%$ [confidence interval $(\mathrm{CI})$ : 0.42 $1.18 \%$ ] reduction per 1-point increment in colonic NEC score, $p<0.001)$. IL-1 $\beta$, IL-8, and IL-10 protein levels in colon were all significantly increased in control animals with severe NEC (scores 5-6) compared with unaffected controls, whereas the colonic IL- $1 \beta$ level in the FMT group was intermediate, and IL-8 and IL-10 levels comparable with control NEC animals (Fig. 4b).

\section{Bacteriology, hematology, and clinical biochemistry}

Oral + rectal FMT treatment increased bacterial colonization of liver and/or spleen on day 5 (89 vs $60 \%, p<0.05, n$ $=18-20$ ), while only few blood samples from days 1,2 , and 3 were culture positive and with no difference between groups. Staphylococci and Enterococci were identified in both groups, but 17 and 39\% of FMT animals were colonized with Escherichia coli and Streptococcus suis (a common pig pathogen), respectively, whereas no control animals were found positive for these species (Suppl. table S3). Additionally, FMT increased total blood leukocytes, neutrophils, and lymphocytes two- to threefold compared with controls on day 5 (all $p<0.05$, Suppl. table S4) with no effect on remaining hematological parameters. Creatinine and $\gamma$-glutamyl transferase, markers of impaired kidney and liver function, were elevated in oral + rectal FMT compared with controls (both $p<0.01$, Suppl. table S4).

\section{Experiment 2: rectal FMT}

\section{Clinical observations}

Three animals could not be resuscitated after birth and died before randomization. In contrast to experiment 1,5 day survival was unaffected by rectal FMT (68 vs $78 \%$ in CON, $n=22-23$, Fig. 1b, right). All animals dying early $(<48 \mathrm{~h})$ presented with sudden respiratory failure with no clinical signs of systemic illness or internal organ pathologies. Similarly, neither of the clinical parameters motor activity and growth rate (Fig. 1b) were affected by rectal FMT. Still, FMT reduced the relative NEC incidence by $75 \%$ when administered rectally (13 vs $50 \%, p<0.05$, Fig. 1b). In this experiment, NEC lesions occurred primarily in the mid or distal small intestine $(6$ vs $40-50 \%$ in CON,$p<0.05$ ), with fewer lesions in stomach (6\%), proximal small intestine (8\%) and colon $(17 \%)$. 
A

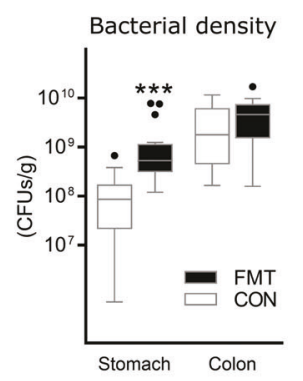

Oral + rectal FMT
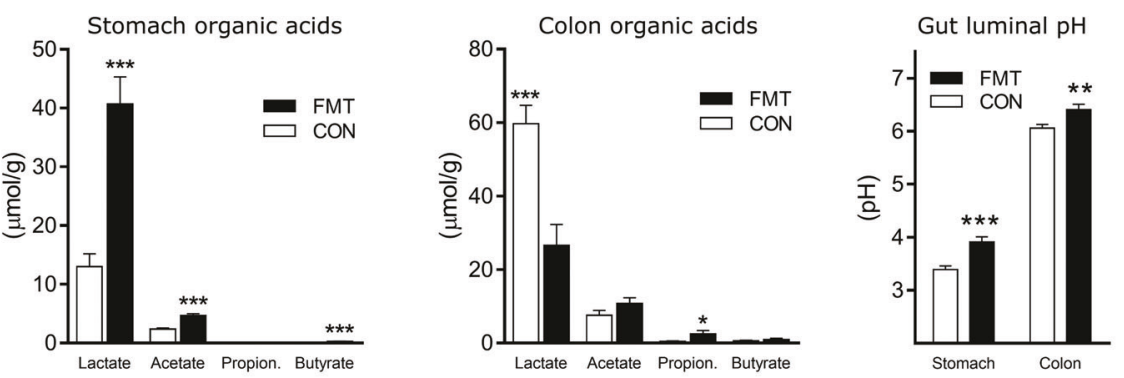

\section{Rectal FMT}
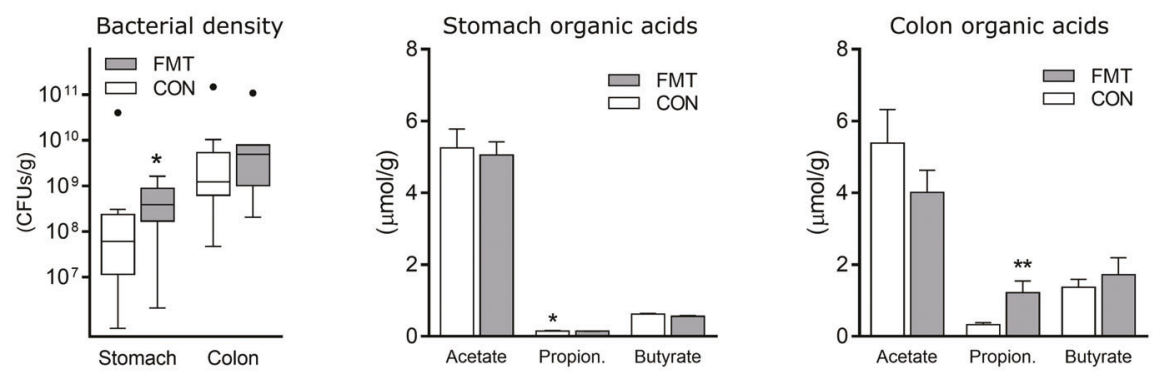

Gut luminal $\mathrm{pH}$

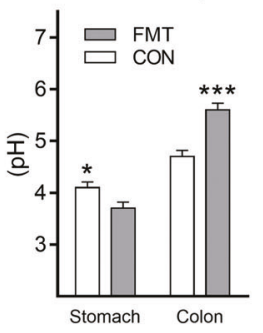

B Small intestinal eubacterial fluorescence in situ hybridisatoin

\section{Oral + rectal FMT}
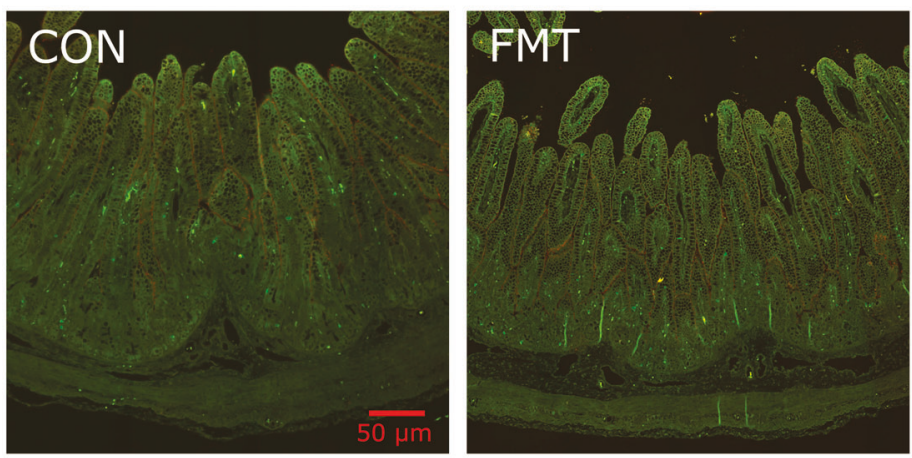

\section{Rectal FMT}

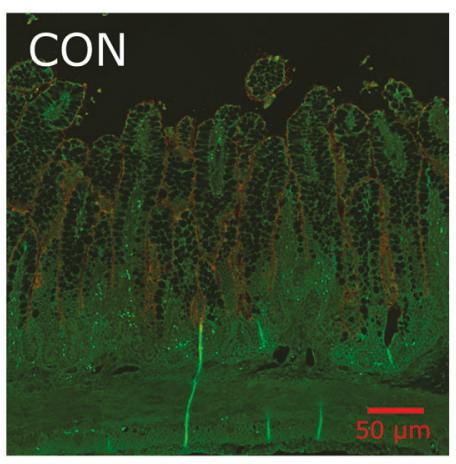

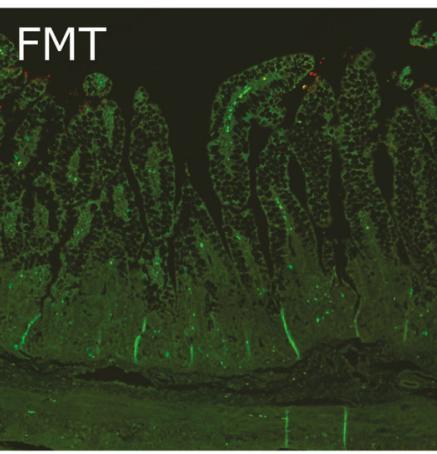

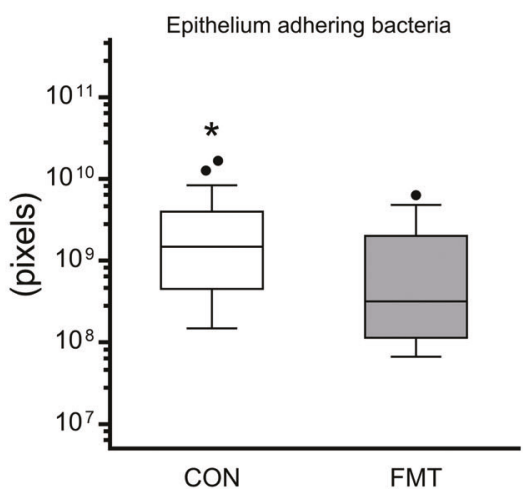


Fig. 3 Gut microbiota metabolism and epithelial adhesion. a Left, gut bacterial densities quantified by qPCR. Center, organic acid levels in stomach and colon content. Right, acidity of stomach and colon luminal content. b Left, representative photomicrographs of fluorescence in situ hybridization stained (eubacterial probe, red fluorochrome signal) small intestinal tissue sections. Right, image analysis-based quantitation of mucosa bacterial adhesion on small intestinal tissue sections. Data are presented as median with interquartile range and statistical tests performed on log-transformed data. $* p<0.05, * * p<0.01, * * * p<0.001$

\section{Gut microbial composition and metabolites}

Like in the previous experiment, rectal FMT treatment changed the bacterial composition in both stomach (unweighted UniFrac, $R=0.816, p<0.001$; weighted UniFrac, $R=0.246, p<0.01$ ) and colon (unweighted UniFrac, $R=0.835, p<0.001$; weighted UniFrac, $R=0.782, p<$ 0.01, Fig. 2a, right). Further, rectal FMT increased the Shannon index of colonic $(p<0.01)$ but not stomach microbiota (Fig. 2b, right) opposite oral + rectal treatment. The stomach bacterial composition resembled that of experiment 1 with Lactobacillus dominance (Fig. 2c). However, rectal FMT animals had a different composition than controls, with greater relative abundance of Lactobacillus ( 89.0 vs $17.6 \%, p<0.001)$, whereas Enterococcus (0.58 vs $24.9 \%, p<0.001)$, Staphylococcus (0.25 vs $19.9 \%$, $p<0.001)$, and Streptococcus $(0.32$ vs $7.63 \%, p<0.01)$ were diminished or absent. In the colon, compositional changes mirrored those in experiment 1 but were more pronounced after rectal FMT, including a major reduction of Enterococcus $(19.4$ vs $82.4 \%, p<0.001)$ as well as introduction of the obligate anaerobe Bacteroides (14.7 vs $0.01 \%, p<0.01)$. Additionally, Lactobacillus (23.8 vs $0.15 \%, p<0.01)$ and Escherichia (27.9 vs $5.83 \%, p<0.05)$ were increased in abundance after FMT treatment. Besides, using PICRUSt functional prediction these compositional changes in the colon after rectal FMT led to enrichment of microbial genes involved in several microbehost signaling pathways. Notably, FMT increased the synthesis of TLR4 ligand lipopolysaccharide, whereas synthesis of TLR2 ligand peptidoglycan was decreased (Suppl. table S5).

Trends in gut bacterial density after rectal FMT resembled those in experiment 1, although the increase in stomach bacterial load was less pronounced $(p<0.05$, Fig. 3a, lower). Unlike oral + rectal FMT, SCFA levels in stomach was minimally affected by rectal FMT treatment (Fig. 3a), and in contrast to experiment 1, stomach $\mathrm{pH}$ was reduced compared with control ( $p<0.05$, Fig. 3a). Further, the experiment confirmed that FMT increased propionate levels $(p<0.01$, Fig. 3a) and luminal $\mathrm{pH}$ in the colon $(p<0.001$, Fig. 3a).

\section{Gut mucosal and systemic responses}

The abundance of mucosa-adhering bacteria in the small intestine was differentially affected by FMT route, and opposite experiment 1 , rectal administration reduced the adherence compared with controls $(p<0.05$, Fig. 3b, lower right). Rectal FMT administration did not affect villus morphometry in any intestinal segment but similar to experiment 1 it increased proximal lactase activity $(p<0.05$, Suppl. table S2) without effects on remaining digestive enzymes or intestinal permeability (pooled median with IQR: 0.05 [0.12], $n=9-11)$. Due to the low colon NEC incidence, mucin density of colonic goblet cells was not affected by rectal FMT (pooled mean with SD: $4.40 \pm 1.9 \%$, $n=14)$. Similarly, the levels of proinflammatory cytokines IL-1 $\beta$ (pooled mean with SD: $1.35 \pm 1.3 \mathrm{ng} / \mathrm{g}, n=16-20$ ) and IL-8 $(1.71 \pm 1.6 \mathrm{ng} / \mathrm{g}, \quad n=16-20)$ were substantially lower than in experiment 1 and with no obvious differences between groups. Interestingly, the leukocyte response was identical to the previous experiment with increased circulating lymphocytes ( $p<0.05$, Suppl. table S4) and neutrophils $(p<0.001)$ following rectal FMT, whereas no markers of internal organ dysfunction (e.g., creatinine, $\gamma$ glutamyl transferase) were elevated.

\section{Discussion}

NEC remains a devastating disease in preterm infants, and the gut microbiota is thought to play a causal role [2]. Preventive strategies include breastfeeding and probiotics administration, while treatment of clinical symptoms is limited to bowel rest, gastric decompression, parenteral antibiotics, and ultimately resection of necrotic intestinal segments with implications for long-term health [31]. Here we describe how NEC-susceptible preterm, cesareandelivered pigs fed infant formula develop a gut dysbiosis resembling that of preterm infants [7, 9], and reflected by low species diversity, high abundance of facultative anaerobic bacteria, and excessive colonic lactate production. In this spontaneous disease model, transplantation of feces from healthy donors profoundly changed the gut bacterial composition in an administration route-dependent manner and protected against NEC. However, our initial investigation into combined oral and rectal FMT administration yielded ambiguous results, as treatment reduced NEC incidence but increased overall mortality, together with gastric bacterial overload, increased bacterial adhesion, and pathogens colonizing internal organs, indicating sepsis as a likely cause of death. These sepsis deaths occurred within $24 \mathrm{~h}$ after FMT treatment, and hence before NEC-onset, which rarely occurs before day 3 of life in preterm pigs. 
A

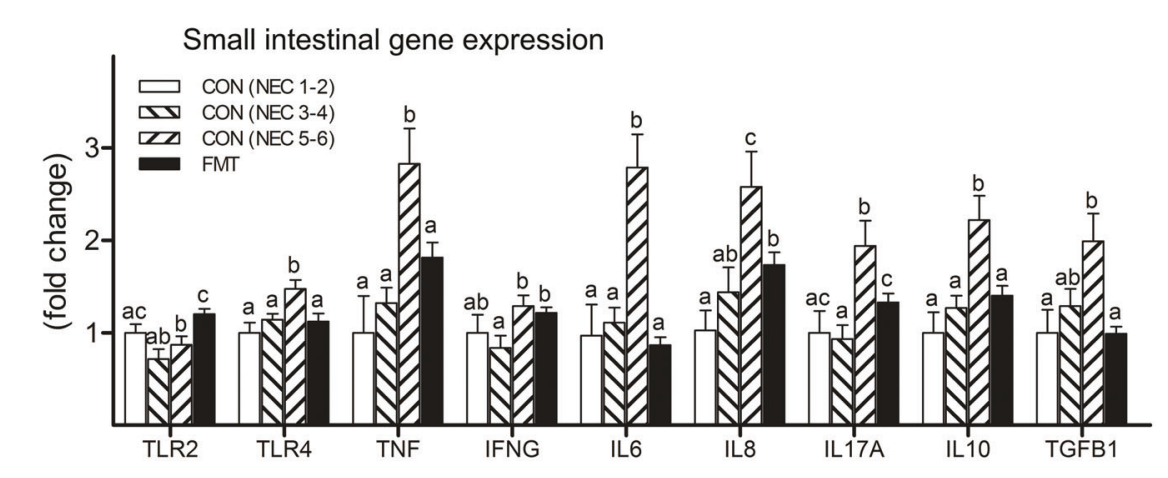

C

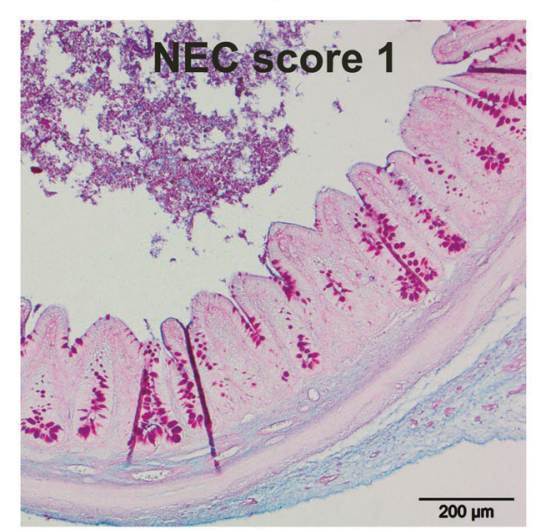

Oral + rectal FMT

B
Alcian blue-periodic acid-Schiff stained colonic goblet cell mucin

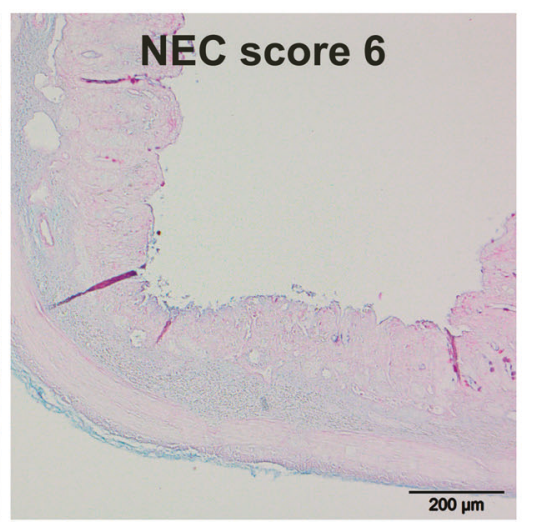

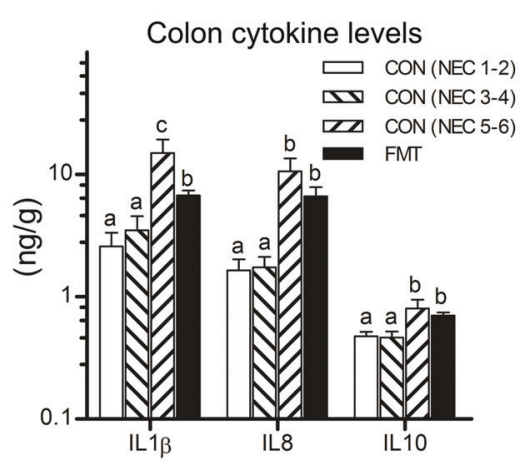

Colon goblet cell mucin density

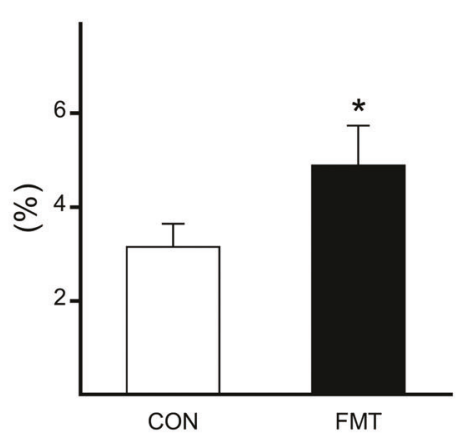

Fig. 4 Gut host response to oral + rectal FMT. a Small intestinal immune gene expression. Control animals were stratified according to their maximum NEC lesion score in any gut segment. Mean expression level for the NEC 1-2 group was set to "1" for all gene assays and remaining groups scaled accordingly. The data were analyzed using a linear mixed model with post hoc tests for pairwise comparisons. Columns not sharing the same letter are significantly different $(p<0.05)$. b Cytokine levels in colon tissue homogenates. The data

Since these two events (sepsis deaths vs NEC diagnosis) were separated in time, we could not decipher whether the reduced NEC incidence on day 5 of life after FMT treatment was merely because the weakest, most susceptible animals had already died. Based on a new hypothesis that oral FMT administration is detrimental to preterm newborns, whereas rectal administration is sufficient to protect from NEC, we performed a follow-up experiment, demonstrating that exclusively rectal FMT could reduce NEC with no negative impact on clinical status, including survival, motor activity, growth, or blood biochemistry. Hence, not only gut colonization patterns but also more importantly the clinical response of preterm newborns to FMT treatment appears to be dependent on administration route, as oral FMT increases the risk of lethal sepsis, whereas rectal FMT protects against NEC without causing adverse effects.

The gut microbiota is transitional and unstable in infants compared with adults [32]. The attainment of a stable microbial composition may be problematic especially for were stratified and analyzed as above. c Left, representative photomicrographs ( $\times 10$ magnification) of Alcian Blue and periodic acidSchiff (AB-PAS)-stained colonic tissue sections visualizing goblet cell-containing acid and neutral mucins from an animal with no NEC lesions (left) and an animal with severe NEC (score 6, right). Right, quantitative measure of goblet cell mucin density by image analysis of AB-PAS-stained colon tissue sections. ${ }^{*} p<0.05$

preterm infants [3], wherein failure to progress from a microbiota dominated by facultative anaerobic bacteria to obligate anaerobes could increase the risk of NEC $[9,10]$. On the other hand, the instability of the neonatal gut microbiota probably means that it is easily replaced by the introduction of a balanced microbial community [32]. In preterm formula-fed pigs, FMT prevented gut dysbiosis and increased the bacterial diversity and relative abundance of obligate anaerobes, reflecting the normal gut microbiota development in infants, including those born extremely preterm [3]. Yet it remains uncertain whether NEC is causally related with overabundance of facultative anaerobes, absence of obligate anaerobes, or a combination. Interestingly, donor feces transplanted only a short distance into the rectum affects proximal parts of the gastrointestinal tract. Hence, rectal FMT increased the relative abundance of Lactobacillus in the stomach, together with lowered luminal $\mathrm{pH}$, which is consistent with NEC protection in preterm infants [33, 34]. In addition, rectal FMT reduced small 
intestinal NEC lesions and bacterial adhesion, indicating retrograde colonization from the distal site of transplantation. FMT has shown similar beneficial effects on intestinal ischemic injury in full term mice administered FMT from juvenile donors into the proximal colon [35], highlighting the redundancy (e.g., disease etiology, recipient gestational age, donor age, and transplantation site) of neonatal FMT treatment.

In the colon, FMT prevented an Enterococcus dysbiosis, excess lactate levels, and an acidic luminal $\mathrm{pH}$, possibly by introducing anaerobic bacteria such as Bacteroides to change the carbohydrate fermentation toward SCFA production and lactate consumption. SCFAs may be important in regulating gut luminal $\mathrm{pH}$ through an epithelial exchange system absorbing SCFAs and secreting bicarbonate [36]. Of note, the colonic microbiota was enriched in genes related to bicarbonate transport following rectal FMT. In preterm infants, NEC is associated with acidic stools (low pH) and elevated urinary lactate of microbial origin [37, 38], whereas the production of SCFA but not lactate by human fecal microbiota is inhibited at low $\mathrm{pH}$ [39]. FMT appears to prevent the depletion of mucin stores in colonic goblet cells that is closely associated with NEC lesion severity. The bacterial load in colon contents of preterm pigs was relatively low and not associated with NEC, supporting

\section{Formula feeding}

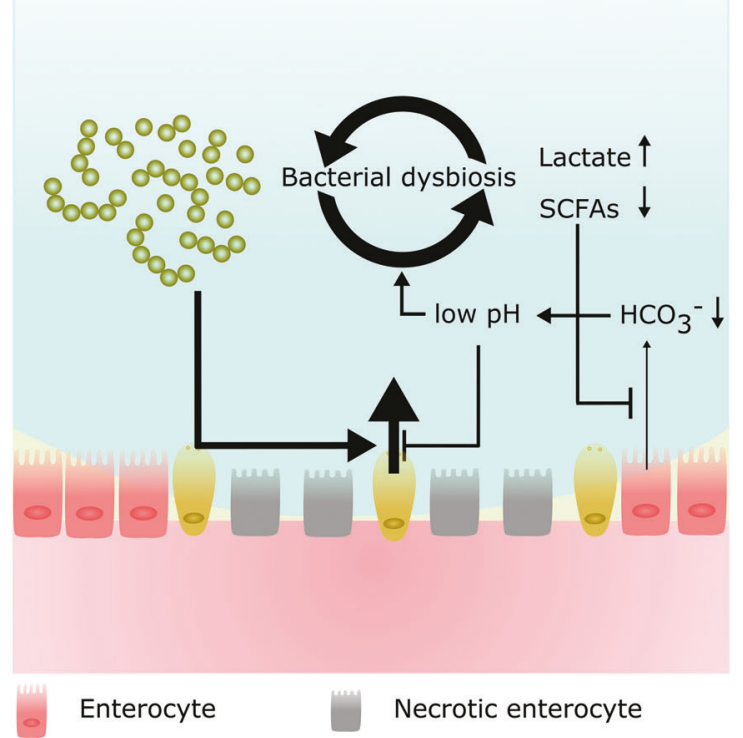

Fig. 5 Conceptual diagram of gut luminal events leading to mucosal damage and NEC, and the prevention by FMT. Left, in preterm newborns, formula feeding favors colonization and growth of facultative anaerobic, lactic acid producing bacteria, leading to lactate overproduction, which in turn lowers the luminal $\mathrm{pH}$ and inhibits the growth of lactate-utilizing bacteria. The lack of SCFA production decreases bicarbonate secretion to the gut lumen. The low luminal $\mathrm{pH}$ inhibits unfolding of the tightly packed mucins rendering the mucus barrier dysfunctional and leaving the mucosa vulnerable to bacterial observations from preterm infants [40], and indicating that the mucin depletion in control animals was unlikely due to excess bacterial stimulation. Instead, we speculate that the acidic colonic lumen of formula-fed preterm newborns inhibits the pH-dependent structural expansion of secreted mucin [41], rendering the mucus barrier dysfunctional and exposing the epithelial surface. Further studies are required to clarify the roles of lactate, SCFAs, and bicarbonate in relation to mucus barrier structure and NEC development (Fig. 5).

Host-microbe interactions in the gut mucosa modulate the host immune response, which is exaggerated in NEC and may involve signaling via the antigen recognition receptor TLR4 [2]. In this study, animals with severe NEC showed increased gene expression of TLR4 and a range of proinflammatory cytokines (e.g., tumor necrosis factor- $\alpha$ (TNF- $\alpha$ ), interferon gamma, interleukin-17) in the small intestine, confirming overt pathological findings on the molecular level. Interestingly, we found that FMT increased intestinal TLR2-TLR4 gene expression ratio with inverse trends in the predicted synthesis of their natural ligands peptidoglycan (decreased) and lipopolysaccharide (LPS) (increased) by the gut microbiota. This could indicate that part of the NEC-protective effect of FMT is due to physiological LPS-TLR4 signaling to acquire antigen tolerance

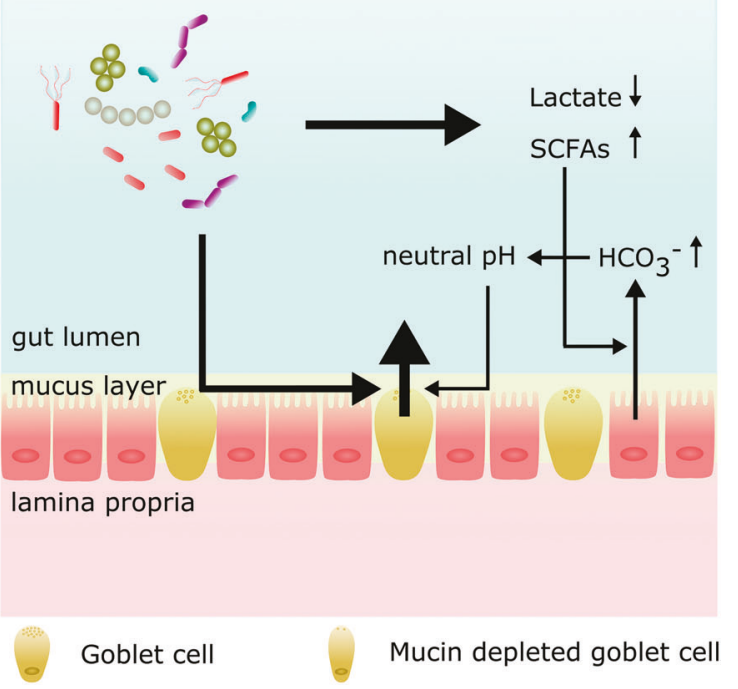

invasion. Right, FMT treatment introduces lactate-utilizing bacteria such as Bacteroides, which changes microbial metabolism towards lactate consumption and SCFA production. The combination of lower lactate and potential exchange of SCFA and bicarbonate ensures a neutral luminal $\mathrm{pH}$. This allows for proper unfolding of secreted mucin, which in turn sustains a physical barrier that is sufficient to protect the epithelial surface from bacterial invasion, thereby preventing mucosal damage and NEC 
[42]. The accuracy of functional prediction by PICRUSt relies on the degree of sequence database mapping, which in this case is high due to sequencing depth adequacy and because the human microbiome database is extensively covered. In the donor feces, we identified several Bacteroides species (e.g., the commensal B. fragilis) that colonized the recipient animals. B. fragilis-specific capsular polysaccharides promote TLR2-dependent gut homeostasis in animal models of colitis [43, 44]. Besides, FMT animals had increased colonic levels of propionate-a biologically active metabolite of anaerobic fermentation shown to promote immune homeostasis in colitis [45]. Hence, FMT may induce gut immune tolerance through altered host-microbe signaling molecules such as bacterial antigens and metabolites.

In addition, we found elevated levels of blood leukocytes following FMT treatment, indicating an effect beyond the gut. Preterm infants and pigs have reduced levels of circulating leukocytes, particularly neutrophils [46], possibly contributing to their increased infection susceptibility. Proper hematopoiesis and infection control depend on a balanced gut microbiota [47], and therefore FMT may act to improve immune cell recruitment, thereby controlling mucosal infection. Detailed effect mechanisms involving microbial antigens or metabolites and host cells in the gut mucosa and bone marrow require further investigation.

Antibiotics are essential medications in neonatal intensive care, and the majority of preterm infants are administered antibiotics during admission, whereby antimicrobial resistance has become a concern. FMT reduces the abundance of antibiotic resistance genes in adult patients [48], but the effect in newborns is unknown. Prolonged use of parenteral antibiotics, often given for other indications than NEC symptoms, is associated with increased risk of NEC [49], as well as enrichment of gut microbial antibiotic resistance genes mapped to Enterobacteriaceae [50]. In preterm infants, preventive treatment with enteral antibiotics protects against NEC, but antimicrobial resistance concerns limit its use in clinical practice [51]. Accordingly, in preterm pigs, short duration enteral but not parenteral antibiotics therapy decreases the gut bacterial load and protects against NEC, but both therapies induce an Enterobacteriaceae dominated dysbiotic state [16]. Possibly, FMT treatment alone or following short-term enteral antibiotics may help control the microbial colonization of preterm newborns and reduce antibiotic resistance.

Short-term, FMT improves bacterial colonization and NEC resistance in preterm pigs, used as a model for preterm infants. Rectal administration is the preferred administration route, as oral administration increases the risk of bacterial translocation and sepsis by exposing the proximal gut to potential pathogens from the donor feces. A longer-term safety assessment of such a pronounce manipulation of spontaneous gut colonization in a delicate population like preterm newborns is desirable. Similarly, further investigation into FMT donor characteristics, screening conditions, refinement of FMT quality, dose, and timing should be completed before clinical testing can be considered.

Acknowledgements We thank Jane Povlsen, Kristina Møller, and Elin Skytte for assistance with animal caretaking and laboratory analyses.

Funding This work was supported by Innovation Fund Denmark and the German Academic Exchange Service.

Data availability Bacterial $16 \mathrm{~S}$ amplicon sequencing data have been deposited in European Nucleotide Archive (accession number: PRJEB26476).

\section{Compliance with ethical standards}

Conflict of interest The authors declare that they have no conflict of interest.

\section{References}

1. Lin PW, Stoll BJ. Necrotising enterocolitis. Lancet . 2006;368:1271-83.

2. Niño DF, Sodhi CP, Hackam DJ. Necrotizing enterocolitis: new insights into pathogenesis and mechanisms. Nat Rev Gastroenterol Hepatol. 2016;13:590-600.

3. La Rosa PS, Warner BB, Zhou Y, Weinstock GM, Sodergren E, Hall-Moore CM, et al. Patterned progression of bacterial populations in the premature infant gut. Proc Natl Acad Sci USA. 2014:111:12522-7.

4. Arboleya S, Binetti A, Salazar N, Fernandez N, Solis G, Hernandez-Barranco A, et al. Establishment and development of intestinal microbiota in preterm neonates. FEMS Microbiol Ecol. 2012;79:763-72.

5. Wang Y, Hoenig JD, Malin KJ, Qamar S, Petrof EO, Sun J, et al. $16 \mathrm{~S}$ rRNA gene-based analysis of fecal microbiota from preterm infants with and without necrotizing enterocolitis. ISME J. 2009;3:944-54.

6. Claud EC, Keegan KP, Brulc JM, Lu L, Bartels D, Glass E, et al. Bacterial community structure and functional contributions to emergence of health or necrotizing enterocolitis in preterm infants. Microbiome. 2013;1:20.

7. Warner BB, Deych E, Zhou Y, Hall-Moore C, Weinstock GM, Sodergren E, et al. Gut bacteria dysbiosis and necrotising enterocolitis in very low birthweight infants: a prospective case-control study. Lancet. 2016;387:1928-36.

8. Taft DH, Ambalavanan N, Schibler KR, Yu Z, Newburg DS, Ward DV, et al. Intestinal microbiota of preterm infants differ over time and between hospitals. Microbiome. 2014;2:36.

9. Pammi M, Cope J, Tarr PI, Warner BB, Morrow AL, Mai V, et al. Intestinal dysbiosis in preterm infants preceding necrotizing enterocolitis: a systematic review and meta-analysis. Microbiome. 2017;5:31.

10. Stewart CJ, Embleton ND, Marrs ECL, Smith DP, Nelson A, Abdulkadir B, et al. Temporal bacterial and metabolic development of the preterm gut reveals specific signatures in health and disease. Microbiome. 2016;4:67.

11. World health organization. Probiotics in Food. Health and nutritional properties and guidelines for evaluation. FAO Food Nutr Pap. 2006;85:2-38. 
12. AlFaleh K, Anabrees J. Probiotics for prevention of necrotizing enterocolitis in preterm infants. Cochrane database Syst Rev. 2014;CD005496.

13. van den Akker CHP, van Goudoever JB, Szajewska H, Embleton ND, Hojsak I, Reid D, et al. Probiotics for Preterm Infants. J Pediatr Gastroenterol Nutr. 2018;67:1.

14. Kelly CR, Kahn S, Kashyap P, Laine L, Rubin D, Atreja A, et al. Update on fecal microbiota transplantation 2015: indications, methodologies, mechanisms, and outlook. Gastroenterology . 2015;149:223-37.

15. Sangild PT, Siggers RH, Schmidt M, Elnif J, Bjornvad CR, Thymann T, et al. Diet- and colonization-dependent intestinal dysfunction predisposes to necrotizing enterocolitis in preterm pigs. Gastroenterology . 2006;130:1776-92.

16. Birck MM, Nguyen DN, Cilieborg MS, Kamal SS, Nielsen DS, Damborg P, et al. Enteral but not parenteral antibiotics enhance gut function and prevent necrotizing enterocolitis in formula-fed newborn preterm pigs. Am J Physiol Gastrointest Liver Physiol. 2016;310:G323-33.

17. Siggers RH, Siggers J, Boye M, Thymann T, Mølbak L, Leser T, et al. Early administration of probiotics alters bacterial colonization and limits diet-induced gut dysfunction and severity of necrotizing enterocolitis in preterm pigs. J Nutr. 2008;138: 1437-44.

18. Cilieborg MS, Thymann T, Siggers R, Boye M, Bering SB, Jensen $\mathrm{BB}$, et al. The incidence of necrotizing enterocolitis is increased following probiotic administration to preterm pigs. $\mathrm{J}$ Nutr. 2011;141:223-30.

19. Sangild PT, Thymann T, Schmidt M, Stoll B, Burrin DG, Buddington RK. Invited review: the preterm pig as a model in pediatric gastroenterology. J Anim Sci. 2013;91:4713-29.

20. Bjornvad CR, Thymann T, Deutz NE, Burrin DG, Jensen SK, Jensen BB, et al. Enteral feeding induces diet-dependent mucosal dysfunction, bacterial proliferation, and necrotizing enterocolitis in preterm pigs on parenteral nutrition. Am J Physiol Gastrointest Liver Physiol. 2008;295:G1092-103.

21. Lagkouvardos I, Joseph D, Kapfhammer M, Giritli S, Horn M, Haller D, et al. IMNGS: a comprehensive open resource of processed 16S rRNA microbial profiles for ecology and diversity studies. Sci Rep. 2016;6:33721.

22. Edgar RC. UPARSE: highly accurate OTU sequences from microbial amplicon reads. Nat Methods. 2013;10:996-8.

23. Edgar RC. Search and clustering orders of magnitude faster than BLAST. Bioinformatics . 2010;26:2460-1.

24. Pruesse E, Peplies J, Glöckner FO. SINA: accurate highthroughput multiple sequence alignment of ribosomal RNA genes. Bioinformatics . 2012;28:1823-9.

25. Lozupone C, Knight R. UniFrac: a new phylogenetic method for comparing microbial communities. Appl Environ Microbiol. 2005;71:8228-35.

26. Caporaso JG, Kuczynski J, Stombaugh J, Bittinger K, Bushman FD, Costello EK, et al. QIIME allows analysis of high-throughput community sequencing data. Nat Methods. 2010;7: 335-6.

27. Langille MGI, Zaneveld J, Caporaso JG, McDonald D, Knights D, Reyes JA, et al. Predictive functional profiling of microbial communities using 16S rRNA marker gene sequences. Nat Biotechnol. 2013;31:814-21.

28. Ellekilde M, Krych L, Hansen CHF, Hufeldt MR, Dahl K, Hansen $\mathrm{LH}$, et al. Characterization of the gut microbiota in leptin deficient obese mice - correlation to inflammatory and diabetic parameters. Res Vet Sci. 2014;96:241-50.

29. Pieper R, Boudry C, Bindelle J, Vahjen W, Zentek J. Interaction between dietary protein content and the source of carbohydrates along the gastrointestinal tract of weaned piglets. Arch Anim Nutr. 2014;68:263-80.
30. Villodre Tudela C, Boudry C, Stumpff F, Aschenbach JR, Vahjen W, Zentek J, et al. Down-regulation of monocarboxylate transporter 1 (MCT1) gene expression in the colon of piglets is linked to bacterial protein fermentation and pro-inflammatory cytokine-mediated signalling. Br J Nutr. 2015;113:610-7.

31. Hintz SR, Kendrick DE, Stoll BJ, Vohr BR, Fanaroff A, Donovan $\mathrm{EF}$, et al. Neurodevelopmental and growth outcomes of extremely low birth weight infants after necrotizing enterocolitis. Pediatrics . 2005; 115:696-703.

32. Lozupone CA, Stombaugh JI, Gordon JI, Jansson JK, Knight R. Diversity, stability and resilience of the human gut microbiota. Nature . 2012;489:220-30.

33. Carrion V, Egan EA. Prevention of neonatal necrotizing enterocolitis. J Pediatr Gastroenterol Nutr. 1990;11:317-23.

34. Terrin G, Passariello A, De Curtis M, Manguso F, Salvia G, Lega L, et al. Ranitidine is associated with infections, necrotizing enterocolitis, and fatal outcome in newborns. Pediatrics. 2012;129:e40-5.

35. Li XX, Li XX, Shang Q, Gao Z, Hao F, Guo H, et al. Fecal microbiota transplantation (FMT) could reverse the severity of experimental necrotizing enterocolitis (NEC) via oxidative stress modulation. Free Radic Biol Med. 2017;108:32-43.

36. Binder HJ, Rajendran V, Sadasivan V, Geibel JP. Bicarbonate secretion: a neglected aspect of colonic ion transport. J Clin Gastroenterol. 2005;39:S53-8.

37. Clark DA, Thompson JE, Weiner LB, McMillan JA, Schneider AJ, Rokahr JE. Necrotizing enterocolitis: intraluminal biochemistry in human neonates and a rabbit model. Pediatr Res. 1985;19:919-21.

38. Garcia J, Smith FR, Cucinell SA. Urinary D-lactate excretion in infants with necrotizing enterocolitis. J Pediatr. 1984;104:268-70.

39. Belenguer A, Duncan SH, Holtrop G, Anderson SE, Lobley GE, Flint HJ. Impact of $\mathrm{pH}$ on lactate formation and utilization by human fecal microbial communities. Appl Environ Microbiol. 2007;73:6526-33.

40. Abdulkadir B, Nelson A, Skeath T, Marrs ECL, Perry JD, Cummings SP, et al. Stool bacterial load in preterm infants with necrotising enterocolitis. Early Hum Dev. 2016;95:1-2.

41. Ambort D, Johansson MEV, Gustafsson JK, Nilsson HE, Ermund A, Johansson BR, et al. Calcium and $\mathrm{pH}$-dependent packing and release of the gel-forming MUC2 mucin. Proc Natl Acad Sci USA. 2012;109:5645-50.

42. Lotz M, Gütle D, Walther S, Ménard S, Bogdan C, Hornef MW. Postnatal acquisition of endotoxin tolerance in intestinal epithelial cells. J Exp Med. 2006;203:973-84.

43. Round JL, Mazmanian SK. Inducible Foxp3+regulatory T-cell development by a commensal bacterium of the intestinal microbiota. Proc Natl Acad Sci USA. 2010;107:12204-9.

44. Round JL, Lee SM, Li J, Tran G, Jabri B, Chatila TA, et al. The Toll-like receptor 2 pathway establishes colonization by a commensal of the human microbiota. Science (80-). 2011;332:974-7.

45. Smith PM, Howitt MR, Panikov N, Michaud M, Gallini CA, Bohlooly-Y M, et al. The microbial metabolites, short-chain fatty acids, regulate colonic Treg cell homeostasis. Science. 2013;341:569-73.

46. Nguyen DN, Jiang P, Frøkiær H, Heegaard PMH, Thymann T, Sangild PT. Delayed development of systemic immunity in preterm pigs as a model for preterm infants. Sci Rep. 2016;6:36816.

47. Khosravi A, Yáñez A, Price JG, Chow A, Merad M, Goodridge HS, et al. Gut microbiota promote hematopoiesis to control bacterial infection. Cell Host Microbe. 2014;15:374-81.

48. Millan B, Park H, Hotte N, Mathieu O, Burguiere P, Tompkins TA, et al. Fecal microbial transplants reduce antibiotic-resistant genes in patients with recurrent Clostridium difficile infection. Clin Infect Dis. 2016;62:1479-86.

49. Cotten CM, Taylor S, Stoll B, Goldberg RN, Hansen NI, Sánchez $\mathrm{PJ}$, et al. Prolonged duration of initial empirical antibiotic 
treatment is associated with increased rates of necrotizing enterocolitis and death for extremely low birth weight infants. Pediatrics. 2009;123:58-66.

50. Gibson MK, Wang B, Ahmadi S, Burnham C-AD, Tarr PI, Warner BB, et al. Developmental dynamics of the preterm infant gut microbiota and antibiotic resistome. Nat Microbiol. 2016;1:16024.

51. Bury RG, Tudehope D. Enteral antibiotics for preventing necrotizing enterocolitis in low birthweight or preterm infants. Tudehope D, editor. Cochrane Database Syst Rev. 2001;CD000405. 